\title{
APLICAÇÃO DE UM INSTRUMENTO DE AUDITORIA EM SERVIÇOS DE FISIOTERAPIA DO SUS NA BAHIA
}

\author{
APPLICATION OF AN AUDITING INSTRUMENT IN \\ PHYSIOTHERAPY SERVICES OF THE SUS IN BAHIA
}

Rizmara do Vale Araújo (Orcid: 0000-0001-7286-3292)

Ítalo Ricardo Santos Aleluia (Orcid: 0000-0001-9499-6360)

Fabiane Costa Santos Fontoura (Orcid: 0000-0003-0537-7512)

Anderson Freitas de Santana (Orcid: 0000-0002-2888-2426)

\section{Contato}

Ítalo Ricardo Santos Aleluia

E-mail: italo.aleluia@ufob.edu.br

Santa Casa de Misericórdia de Salvador, Bahia, Brasil.

Centro das Ciências Biológicas e da Saúde, Universidade Federal do Oeste da Bahia, Bahia, Brasil.

${ }^{3}$ Secretaria de Saúde do Estado da Bahia, Bahia, Brasil

\section{RESUMO}

O objetivo deste estudo foi aplicar um instrumento de auditoria em fisioterapia, para analisar o grau de conformidade em dois serviços ambulatoriais de fisioterapia do SUS na Bahia. Realizou-se um estudo de caso com análise de dois serviços: um da rede própria e outro contratualizado. Utilizaram-se a análise documental, a observação não participante, a entrevista com informantes-chave e o registro em diário de campo. Os serviços foram analisados a partir de um escore simples para cada critério pertencente às seguintes dimensões: verificação normativa, estrutura física, gestão de pessoas, recursos materiais, organização, funcionamento e gestão de resultados. Ambos os serviços foram classificados com grau de conformidade incipiente, por não atenderem a, pelo menos, 50\% dos critérios de conformidade exigidos no instrumento de auditoria aplicado. No serviço da rede própria, as dimensões com maior inconformidade corresponderam à adequação normativa ( 0 do total de 5 pontos), aos recursos materiais ( 1 do total de 8 pontos) e à gestão de resultados (3 do total de 13 pontos). Já no serviço contratualizado pelo SUS, as maiores inconformidades foram evidenciadas na gestão de pessoas ( 5 do total de 16 pontos), organização e funcionamento (3 do total de 17 pontos) e gestão de resultados (1 do total de 13 pontos). Novos estudos sobre auditoria em fisioterapia no SUS são necessários em cenários municipais e estaduais, bem como a formulação de novos instrumentos que atualizem dimensões e critérios de análise para refinar estratégias metodológicas nas auditorias de fisioterapia do SUS. Palavras-chave: Auditoria médica; Sistema único de saúde; Fisioterapia; Serviços de saúde.

\footnotetext{
ABSTRACT

The aim of this study was to apply the proposal of an auditing instrument in physiotherapy, to analyse the degree of compliance in two outpatient physiotherapy services of the SUS in a municipality of Bahia. A case study was conducted with in-depth analysis of two services: one from the own network and the other contracted. We used documentary analysis, non-participant observation, interview with key informants and field diary recording. The services were intensively analyzed and are assigned from a simple score for each criterion belonging to the following dimensions: normative verification, physical structure, people management, material resources, organization and operation, and results management. Both services were classified as incipiently compliant because they did not meet at least $50 \%$ of the compliance criteria required in the auditing instrument applied. The services did not achieve minimum scores compatible with an adequate degree of compliance. The service of the own network reached a score below $50 \%$ of the predicted score. In the service of the own network, the dimensions with the greatest non-conformity corresponded to the normative adequacy ( 0 of the total of 5 points), the material resources ( 1 of the total of 8 points), and the management of results ( 3 of the total of 13 points). The situation was similar in the private service, except in the normative adequacy and physical structure, where the percentage was higher than $70 \%$ of the total predicted. The greatest non-conformities in the public service corresponded to the normative adequacy $(0 \%)$, material resources (18.7\%) and results management (22.7\%). In the service contracted by The SUS, the greatest non-conformities were evidenced in the management of people ( 5 of the total of 16 points), organization and functioning ( 3 of the total of 17 points), and results management (1 of the total of 13 points). Further studies on physical therapy auditing in the SUS are necessary in municipal and state scenarios, as well as the formulation of new instruments that update dimensions and analysis criteria to refine methodological strategies in audits of the SUS' physiotherapy. Keywords: Medical Audit; Unified Health System; Physical therapy specialty; Health services.
} 


\section{INTRODUÇÃO}

A auditoria em saúde tem como objetivo avaliar a eficiência e a eficácia de serviços. No Sistema Único de Saúde (SUS), essa atividade busca cumprir as regulamentações das leis, analisando o funcionamento do sistema e serviços de saúde, para prevenir gastos desnecessários, fraudes, corrigir problemas existentes, priorizando o controle e a qualidade do atendimento aos usuários ${ }^{1}$.

Os serviços de fisioterapia no SUS estão enquadrados como atenção especializada; e o Brasil possui cerca de 20.100 serviços, entre próprios e contratualizados². Somente em 2011, a Bahia já registrava um quantitativo de 1.120 serviços, distribuídos em 417 municípios e 9 regiões de saúde ${ }^{3}$.

A velocidade de crescimento dos atendimentos e custos com serviços públicos de fisioterapia são fatores que justificam um controle maior e mais específico na auditagem daqueles que integram o SUS ${ }^{4}$. Em 2008, os atendimentos de fisioterapia ambulatorial no SUS totalizaram, aproximadamente, mais de 36 milhões de sessões, sendo que, em 2014, foram registradas mais de 44 milhões, representando um aumento da ordem de 5,9\% na produção dos serviços'. Os custos com atendimentos revelaram valores aprovados significativos para o ano de 2008 (mais de R\$ 180 milhões); e, em 2014, alcançaram mais de R\$232 milhões (crescimento de aproximadamente $4,5 \%)^{4}$.
A auditagem de serviços públicos de saúde sempre esteve circunscrita a categorias de médicos e enfermeiros, e isso persiste na atualidade. A auditoria estadual de fisioterapia no SUS da Bahia, por exemplo, é realizada por profissionais de outras áreas sem a participação do profissional fisioterapeuta ${ }^{5}$, com fragilidades no processo de trabalho dos auditores, dada a falta de conhecimentos específicos sobre os equipamentos, procedimentos e estrutura mínima dos serviços fisioterapêuticos ${ }^{3}$. Esse fato se torna mais problemático em municípios de pequeno e médio porte, afastados dos grandes centros urbanos, onde a implantação do componente municipal de auditoria é bastante incipiente, o que nos leva a questionar a verdadeira situação do controle exercido sobre os prestadores.

Diante da necessidade de ampliar o escopo de ações da auditoria do SUS, defende-se a composição de equipes multiprofissionais, com participação de outros núcleos de saber ${ }^{3}$. O Conselho Federal de Fisioterapia e Terapia Ocupacional (Coffito) reconhece que o fisioterapeuta pode desempenhar atividades de auditoria, atribuição ratificada em 2012, em resolução específica sobre o tema ${ }^{6}$.

As auditorias de fisioterapia devem avaliar a eficiência e a eficácia dos serviços. Corresponde a uma análise cuidadosa e sistemática das práticas, com o intuito de identificar possíveis erros éticos, morais, entre outros, analisar os parâmetros exigidos de eficiência, eficácia 
e de efetividade, de forma sigilosa, clara e com autonomia, por meio de relatórios com sugestões para possíveis ajustes do serviço, qualificando, assim, a assistência prestada $^{6}$.

A insuficiência de instrumentos de auditoria em fisioterapia na literatura aponta para um cenário desafiador no exercício do controle e da regulação dos serviços públicos, pelos entes municipais e estaduais ${ }^{7}$. Alguns trabalhos vêm propondo instrumentos de auditoria com diferentes focos de análise $e^{5,8}$.

$\mathrm{Na}$ revisão de literatura deste trabalho, foram encontradas três propostas de instrumentos de auditoria para os serviços fisioterapêuticos, que variaram de propostas de avaliação da qualidade e assistência prestada conforme a percepção dos usuários a protocolos destinados aos serviços públicos no que tange aos aspectos normativos, de estrutura, processos e resultados ${ }^{7,8}$. Contudo, nenhuma investigação direta sobre os serviços foi encontrada em nível nacional e internacional, que denotasse a utilização de instrumentos para estimativa do grau de conformidade no setor público.

Este estudo teve como objetivo aplicar a proposta de um instrumento de auditoria em fisioterapia, para analisar o grau de conformidade em dois serviços ambulatoriais de fisioterapia do SUS na Bahia.

\section{MATERIAIS E MÉTODOS}

Adotou-se como estratégia metodológica o estudo de caso, cuja aplicação do instrumento de auditoria ocorreu em dois serviços de fisioterapia ambulatorial: um da rede própria e outro contratualizado para prestar atendimento ao SUS, em município do estado da Bahia. A escolha dos dois serviços com configurações administrativas distintas (próprio e contratualizado) foi intencional, para compreender como o instrumento se aplicaria a contextos organizacionais diferentes, haja vista que os serviços de fisioterapia do SUS estão, em sua maioria, concentrados no setor privado.

A seleção dos serviços participantes do estudo se baseou em critérios distintos. No serviço próprio, considerouse estar implantado há, pelo menos, um ano; ofertar atendimento de fisioterapia ambulatorial; possuir responsável técnico pelo serviço; constar registro no Cadastro Nacional de Estabelecimentos de Saúde (CNES); e possuir capacidade instalada de, no mínimo, 12 usuários profissional/ dia, conforme orienta a resolução de parâmetros assistenciais do Coffito. No serviço privado, além dos critérios anteriores, considerou-se a existência de, pelo menos, um ano de contratualização com o SUS. Um dos serviços pré-selecionado pelos critérios anteriores foi excluído da pesquisa em função de o coordenador ter se recusado a participar do estudo.

Para seleção dos serviços, aplicamos os critérios citados, obedecendo às seguintes etapas: consulta preliminar, no CNES, daqueles existentes no município e vinculados ao SUS, em que foram analisadas informações sobre o tipo de estabelecimento (se ambulatorial ou hospitalar), a esfera administrativa (se federal, estadual ou municipal) e o tipo de prestador (se da administração direta, privada ou outro). A partir dos critérios de seleção dos 
serviços, os pesquisadores validaram as informações obtidas no CNES, mediante consulta à gestão municipal, para escolha final dos estabelecimentos que participariam do estudo.

O serviço contratualizado pelo SUS correspondeu a uma clínica de especialidades médicas, de natureza privada e que ofertava atendimento ambulatorial de fisioterapia, mediante financiamento e gestão contratual do município. Quatro fisioterapeutas que atendiam usuários referenciados por uma Central de Regulação Municipal (CRM) faziam parte do corpo profissional. Quanto ao serviço da rede própria municipal, tratou-se uma unidade ambulatorial para atendimento da demanda espontânea de fisioterapia, constituído por seis fisioterapeutas e sob gestão direta municipal.

A produção dos dados desta pesquisa ocorreu entre setembro e outubro de 2018. O instrumento de auditoria em fisioterapia aplicado foi adaptado a partir da proposta de Aleluia e Santos ${ }^{5}$. As dimensões de análise abarcavam as seguintes questões: adequação normativa, estrutura física dos serviços, gestão de pessoas, recursos materiais, organização e funcionamento e gestão de resultados. Essas dimensões consolidavam critérios de avaliação organizados em escore simples de pontuação, em que a presença de critério nos serviços analisados pontuava um, e a ausência pontuava zero, totalizando 80 pontos. Ressaltamos que o instrumento utilizado nesta pesquisa passou por processo de validação com 16 especialistas (auditores do SUS), por meio do ciclo Delphi, em três rodadas, de forma individual, anônima e eletrônica.
$\mathrm{Na}$ análise do grau de conformidade dos serviços, os pesquisadores atribuíram cumprimento (existência) ou não cumprimento (ausência) de cada critério nos serviços, a partir do cotejamento das evidências presentes na análise documental, na observação in loco e na entrevista semiestruturada com o coordenador do serviço. As entrevistas abordaram perguntas relativas aos critérios propostos. Os documentos e a observação foram guiados por roteiros elaborados pelos pesquisadores, com itens passíveis de comprovação documental ou observacional. Os respectivos roteiros funcionaram como guias, para que os pesquisadores se cercassem de um maior número de fontes de evidências possíveis, na classificação quanto ao cumprimento (ou não) de cada critério.

Nas situações em que os critérios do instrumento não se aplicavam à realidade dos serviços, tais critérios não foram considerados na análise, e foi realizada uma redistribuição da pontuação final do instrumento, excluindo-os e recalculando o escore total, por meio de uma regra de três simples. A tabela 1 sumariza cada dimensão, critério, pontuação de cada dimensão e o total de pontos do instrumento, além de indicar a correspondência de cada critério e suas respectivas fontes de evidência para atribuir a pontuação, seja entrevista, observação, análise documental, seja a combinação de uma ou mais dessas fontes. 
Tabela 1. Dimensões e critérios utilizados para análise do grau de conformidade nos serviços investigados do estudo

\begin{tabular}{|c|c|c|}
\hline Dimensão & Critérios & Pontuação \\
\hline \multirow{15}{*}{$\begin{array}{l}\text { Adequação Nor- } \\
\text { mativa }\end{array}$} & Existência de registro de pessoa jurídica em cartório ${ }^{* *}$ & 1 \\
\hline & Existência de alvará de funcionamento emitido pela prefeitura ${ }^{* *}$ & 1 \\
\hline & Alvará de funcionamento em local de fácil visibilidade* & 1 \\
\hline & Alvará de funcionamento com prazo de validade regular** & 1 \\
\hline & Existência de alvará sanitário ${ }^{* *}$ & 1 \\
\hline & Alvará sanitário em local de fácil visibilidade ${ }^{*}$ & 1 \\
\hline & Alvará sanitário com prazo de validade regular ${ }^{\star *}$ & 1 \\
\hline & Existência de registo no órgão de classe competente ${ }^{\star *}$ & 1 \\
\hline & $\begin{array}{l}\text { Existência de Declaração de Regularidade de Funcionamento (DRF) válida e em } \\
\text { local visível }^{\star \star}\end{array}$ & 1 \\
\hline & Existência de anotação de responsabilidade técnica $(\mathrm{RT})^{\star \star}$ & 1 \\
\hline & $\begin{array}{l}\text { Existência de fisioterapeuta responsável técnico devidamente registrado no órgão } \\
\text { de classe }^{\star \star}\end{array}$ & 1 \\
\hline & Existência de Plano de Gerenciamento de Resíduos Sólidos (PGRSS)** & 1 \\
\hline & $\begin{array}{l}\text { Cadastrado atualizado no Cadastro Nacional de Estabelecimentos de Saúde } \\
(\mathrm{CNES})^{\star \star}\end{array}$ & 1 \\
\hline & Existência de certificado de dedetização atual ${ }^{\star \star}$ & 1 \\
\hline & Apresentação do contrato/convênio que estabelece o vínculo com SUS ${ }^{* *}$ & 1 \\
\hline Subtotal & & 15 \\
\hline \multirow{11}{*}{ Estrutura Física } & Existência de acessibilidade arquitetônica para pessoas com deficiência* & 1 \\
\hline & Existência de extintores de incêndio com prazo de validade regular ${ }^{\star}$ & 1 \\
\hline & $\begin{array}{l}\text { Ambiente físico comporta capacidade de comportar a realização dos procedimentos } \\
\text { cadastrados }^{*}\end{array}$ & 1 \\
\hline & Existência de sala de espera ou recepção* & 1 \\
\hline & Existência de espaço exclusivo destinado à administração do serviço* & 1 \\
\hline & Existência de salas exclusivas para exames e avaliações fisioterapêuticas ${ }^{\star}$ & 1 \\
\hline & Existência de banheiros separados por sexo* & 1 \\
\hline & Banheiros adaptados para pessoas com deficiência ${ }^{*}$ & 1 \\
\hline & Ambiente em geral em boas condições de higienização* & 1 \\
\hline & Ambiente em geral em boas condições de temperatura* & 1 \\
\hline & $\begin{array}{l}\text { Todas as dependências do serviço bem sinalizadas e de fácil identificação para o } \\
\text { usuário* }^{*}\end{array}$ & 1 \\
\hline Subtotal & & 11 \\
\hline \multirow{8}{*}{ Recursos Materiais } & O serviço atende aos equipamentos mínimos ${ }^{*}$ & 1 \\
\hline & $\begin{array}{l}\text { Existência da listagem/registro de todos os equipamentos, materiais e insumos uti- } \\
\text { lizados }^{* *}\end{array}$ & 1 \\
\hline & Cadastro atualizado de materiais e equipamentos no $\mathrm{CNES}^{* *}$ & 1 \\
\hline & Todos os materiais e equipamentos em boas condições de uso* & 1 \\
\hline & Existência de contrato de manutenção corretiva e preventiva ${ }^{* *}$ & 1 \\
\hline & Disponibilidade de materiais ou equipamentos para substituição emergencial ${ }^{*}$ & 1 \\
\hline & $\begin{array}{l}\text { Existência de acesso físico ou online de todos os manuais dos equipamentos utili- } \\
\text { zados }^{* *}\end{array}$ & 1 \\
\hline & $\begin{array}{l}\text { Existência de condições de armazenamento adequada e segura dos materiais e equi- } \\
\text { pamentos* }^{*}\end{array}$ & 1 \\
\hline
\end{tabular}




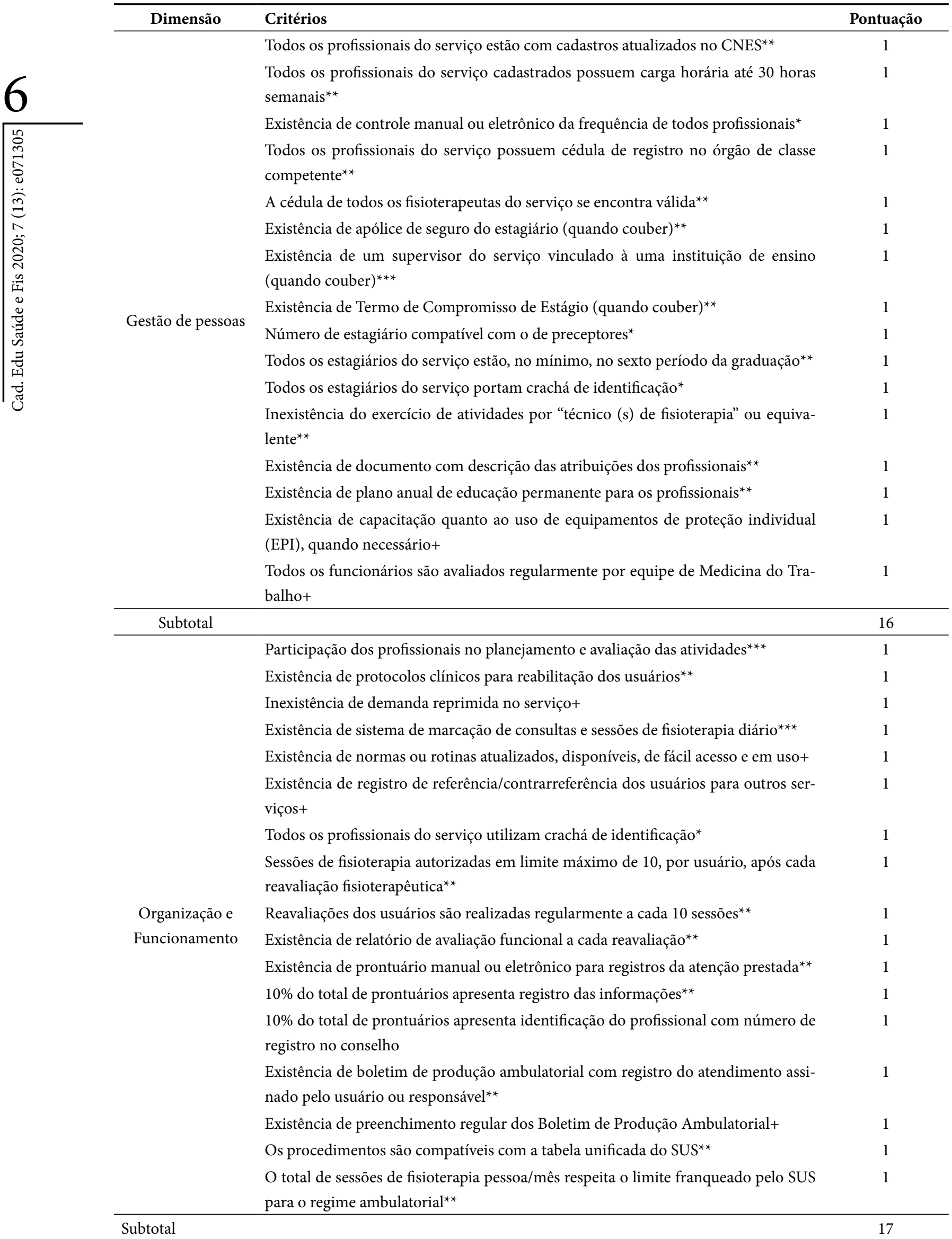




\begin{tabular}{|c|c|c|}
\hline Dimensão & Critérios & Pontuação \\
\hline \multirow{13}{*}{$\begin{array}{l}\text { Gestão de Resul- } \\
\text { tados }\end{array}$} & Profissionais com desempenho avaliado regularmente ${ }^{+}$ & 1 \\
\hline & Profissionais com avaliação regular da satisfação com o trabalho ${ }^{+}$ & 1 \\
\hline & $\begin{array}{l}\text { Existência de metas estabelecidas pelo serviço e/ou estipuladas no contrato ou } \\
\text { convênio com o SUS }\end{array}$ & 1 \\
\hline & Existência de meios de monitoramento de metas ${ }^{+}$ & 1 \\
\hline & $\begin{array}{l}\text { Existência de meios de comunicação com usuários sobre suas reclamações, su- } \\
\text { gestões e solicitações }\end{array}$ & 1 \\
\hline & Usuários com satisfação regularmente avaliada pelo serviço ${ }^{+}$ & 1 \\
\hline & $\begin{array}{l}\text { Existência de reuniões regulares de reunião de equipe comprovadas mediante } \\
\text { apresentação de } \operatorname{atas}^{+}\end{array}$ & 1 \\
\hline & O serviço dispõe de indicadores e os utiliza na avaliação de seus resultados ${ }^{+}$ & 1 \\
\hline & $\begin{array}{l}\text { Resultados das avaliações de satisfação divulgados e discutidos com os profissio- } \\
\text { nais do serviço }{ }^{* * *}\end{array}$ & 1 \\
\hline & Resultados das avaliações de satisfação divulgados para os usuários ${ }^{* * *}$ & 1 \\
\hline & Existência de relatórios com o registro da produção mensal ${ }^{\star \star}$ & 1 \\
\hline & $\begin{array}{l}\text { Coerência entre os procedimentos faturados e o registro do Boletim de Produção } \\
\text { Ambulatorial }^{\star *}\end{array}$ & 1 \\
\hline & $\begin{array}{l}\text { Produção ambulatorial compatível com os parâmetros assistenciais (considerar a } \\
\text { especialidade do atendimento e o perfil clínico do usuário)** }\end{array}$ & 1 \\
\hline Subtotal & & 13 \\
\hline Total & & 80 \\
\hline
\end{tabular}

*Critério validado em observação; ${ }^{* *}$ Critério validado na análise documental; ${ }^{* *}$ Critério validado na entrevista com responsável técnico do serviço; +Critério validado na observação, análise documental e entrevista com responsável técnico

A classificação final do grau de conformidade entre os dois serviços analisados considerou o alcance de pontos em relação ao total previsto no instrumento. Essa classificação foi expressa em percentual e conforme proposta por Aleluia e colaboradores ${ }^{7}$ : grau de conformidade incipiente $(\leq 50 \%$ do total de pontos previsto), conformidade intermediária $(\geq 50 \% \mathrm{e}$ $\leq 70 \%)$ e conformidade adequada $(\geq 70 \%)$.

Tanto os coordenadores dos serviços participantes desta pesquisa quanto o gestor municipal assinaram um termo de anuência institucional autorizando sua realização, e houve submissão do projeto deste estudo ao Comitê de Ética em Pesquisa da Facul- dade de Tecnologia e Ciências (FTCSalvador/Ba), mediante aprovação $\mathrm{n}^{\circ}$ 024939/2015, atendendo às disposições da resolução 466/2012.

\section{RESULTADOS}

Os serviços pesquisados pertenciam a um município de pequeno porte, localizado a $210 \mathrm{~km}$ da capital baiana, com extensão territorial de $1.016,006 \mathrm{~km}^{2}$, população estimada de 68.146 habitantes e IDH de 0,61. Durante o período da produção dos dados, a rede de saúde municipal possuía seis serviços de fisioterapia, sendo um público e os demais privados contratualizados pelo SUS. 
Ambos os serviços foram classificados com grau de conformidade incipiente, por não atenderam a, pelo menos, $50 \%$ dos critérios de conformidade exigidos no instrumento de auditoria aplicado.

No serviço da rede própria, as dimensões com maior inconformidade eram a adequação normativa (0 do total de 5 pontos), os recursos materiais (1 do total de 8 pontos) e a gestão de resultados (3 do total de 13 pontos). Já no serviço contratualizado pelo SUS, as maiores inconformidades foram evidenciadas na gestão de pessoas ( 5 do total de 16 pontos), organização e funcionamento (3 do total de 17 pontos) e gestão de resultados (1 do total de 13 pontos).

Nas questões normativas, ambos os serviços não mantinham atualizados dados no CNES, sobre profissionais ativos e desligados, suas respectivas cargas horárias e sobre os equipamentos em uso nos atendimentos fisioterapêuticos. Chamou a atenção, no serviço da rede própria, a ausência de alvará sanitário, anotação de responsabilidade técnica e registro no Conselho Regional de Fisioterapia e Terapia Ocupacional da Bahia.

A principal inconformidade sobre a estrutura física dos serviços era o não atendimento às prerrogativas legais para garantia da acessibilidade arquitetônica para pessoas com deficiência. $\mathrm{O}$ serviço da rede própria, em especial, não possuía espaço exclusivo para administração do serviço, exames ou avaliações fisioterapêuticas; inexistiam banheiros separados por sexo e sinalização visual e tátil em todas as dependências, além de não contar com itens de segurança básica, como de extintores de incêndio.
As condições de armazenamento dos materiais e equipamentos em ambos os serviços estavam adequadas, e havia acesso tanto físico quanto eletrônico a todos os manuais dos equipamentos. Entretanto, constatou-se que, em ambos os casos, tais equipamentos não estavam cadastrados no CNES e não atendiam à listagem daqueles considerados "mínimos" pela legislação estadual do SUS-Bahia, para o funcionamento adequado de um serviço de fisioterapia.

A manutenção corretiva e preventiva dos equipamentos foi um ponto importante de inconformidades. No serviço da rede própria municipal, essa situação se tornou ainda mais problemática em função da existência de equipamentos danificados e sem controle, pela gestão do serviço, daqueles em funcionamento e dos que possuíam necessidade de manutenção corretiva e preventiva.

O número de profissionais atuantes em ambos os serviços não correspondia ao total de profissionais cadastrados no CNES. Havia disparidade entre a realidade dos estabelecimentos e o CNES, sobretudo quanto ao quantitativo de profissionais ativos e desligados. Alguns dos profissionais não portavam a cédula do registro profissional no momento da observação in loco dos pesquisadores.

Um dos pontos mais críticos foi a gestão de pessoal nos serviços pesquisados. Inexistiam ações de educação permanente regular e protocolos com a definição das atribuições dos profissionais. O serviço da rede própria municipal se destacou quanto ao controle regular da frequência dos colaboradores, ainda que manual. 
Houve diferenças importantes na organização e funcionamento entre os serviços pesquisados. Apenas o serviço privado possuía um sistema de marcação de sessões de fisioterapia informatizado e integrado com a Central Municipal de Regulação (CMR), enquanto, na rede própria, as marcações ocorriam de forma manual e desarticulada do serviço de regulação municipal. Em ambos os estabelecimentos, não se evidenciou o uso de protocolos de reabilitação, tampouco havia fluxo de usuários regulado sob a lógica da referência e contrarreferência, com serviços primários de atenção à saúde. Nenhum instrumento para esse fim foi referido pelos coordenadores de serviço ou evidente na análise documental. Mesmo com a integração de um deles ao sistema de regulação, a marcação de sessões fisioterapêuticas ocorria em uma lógica de programação, isto é, mediante a autorização a cada dez sessões concluídas, talvez desconsiderando as necessidades e singularidades de cada usuário da rede de atenção.

O serviço privado se destacou pela inconformidade nos registros dos atendimentos fisioterapêuticos. Inexistiam prontuários, e os poucos registros evidentes eram realizados em uma ficha com informações sobre a descrição da queixa principal, as condutas, o nome e a assinatura do profissional executante. Esse fato foi diferente no serviço da rede própria que, além dos prontuários, contava com boletins de registro da produção ambulatorial com data e assinatura do usuário.

Houve indícios incipientes de gestão de resultados entre os serviços analisados. Observou-se que ambos apresentaram comprovação documental do número de procedimentos cobrados, mas não monitoravam regularmente a evolução clínica dos usuários, por meio de reavaliações documentadas e relatórios de alta. No serviço público, existia um frágil canal de comunicação com usuários, restrito a uma "caixa de sugestões", porém, sem qualquer evidência de sua utilização para reorientação das práticas de saúde e de gestão do serviço. Não foram encontradas incompatibilidades entre a produção dos serviços e os parâmetros assistenciais vigentes.

Por fim, a gestão participativa demonstrou ser um ponto crítico nos serviços pesquisados, uma vez que usuários e profissionais não participavam de debates para compor ações de planejamento participativo e avaliação das ações e serviços prestados.

\section{DISCUSSÃO}

A aplicação do instrumento se mostrou potente para análise do grau de conformidade dos serviços de fisioterapia próprios e contratualizados pelo SUS, por meio da identificação dos principais pontos críticos e de potencialidades.

As divergências cadastrais entre a realidade dos serviços e o CNES podem fragilizar processos decisórios dos gestores e a formulação de estratégias para qualificação dos serviços de fisioterapia que compõem o sistema municipal de saúde. A atualização cadastral dos serviços de saúde é fundamental para subsidiar auditorias na verificação da veracidade na relação entre serviços programados e executados. São indispensáveis, portanto, estratégias de monitoramento dos dados cadastrais no CNES, principalmente para aqueles contratualizados, uma vez que se trata de uma relação de contraprestação9. 
Manter a atualização de dados cadastrais no CNES é imprescindível para a gestão do sistema e serviços de saúde, pois garante a disponibilidade de informações para subsidiar o planejamento em saúde e o controle social sobre os serviços, seja por meio da ação popular direta, seja pelos órgãos de controle e auditoria interno e externo. A atualização das informações do CNES também é útil à regulação, à avaliação, ao controle, à auditoria e ao ensino/pesquisa, o que torna fundamental a garantia de correspondência entre os dados cadastrais e a realidade dos serviços ${ }^{10}$.

A desatualização de dados do CNES evidente em nosso estudo se assemelhou à realidade de serviços de fisioterapia na capital baiana ${ }^{11}$. Essa incompatibilidade entre os dados cadastrais e a realidade dos serviços ratifica a pouca confiabilidade desses dados para auditores e gestores do SUS utilizarem informações no processo de planejamento e controle social em saúde no âmbito local, o que contraria os propósitos da utilidade pública do CNES, enquanto canal de transparência para divulgação de informações sobre serviços de saúde.

Além do CNES, não há um sistema de registro de equipamentos nos serviços de saúde em nível federal, que, diante das inconsistências encontradas nos serviços do nosso estudo, representa uma importante fragilidade para a gestão municipal monitorar necessidades estruturais dos serviços de fisioterapia do SUS 9 .

Um dado importante foi referente à predominância de serviços de fisioterapia contratualizados pelo SUS, no município, o que exige da auditoria municipal um maior controle sobre as ações ofertadas. Essa realidade tem sido semelhante à de outras regiões de saúde no estado da Bahia, onde os serviços ambulatoriais de fisioterapia do SUS estão concentrados no setor privado contratualizado ${ }^{7}$.

A concentração de serviços de fisioterapia contratualizado pelo SUS no estado da Bahia desperta um sinal de alerta, já que a qualidade dos serviços prestados e o cumprimento das prerrogativas legais, nos estabelecimentos, dependem, também, da ação coordenada entre os componentes municipais e estaduais de auditoria do SUS. O controle exercido por esses entes federativos é de suma importância para um adequado monitoramento dos custos, da produção e da qualidade da atenção prestada, por se tratar de uma relação de contraprestação, em que interesses de mercado podem se tornar alheios aos interesses públicos.

Os registros de carga horária encontrados no sistema de informação se revelaram superiores (40 horas semanais) ao preconizado na legislação do Coffito, contrariando prerrogativas legais do Conselho, que assegura carga horária máxima de 30 horas semanais para fisioterapeutas tanto no âmbito ambulatorial quanto no hospitalar.

As inconformidades encontradas sobre a inexistência de alvará sanitário no serviço público ferem requisitos básicos para o funcionamento seguro dos serviços de saúde, como alvará de funcionamento e alvará sanitário. $\mathrm{O}$ atendimento a essas prerrogativas são fundamentais para proporcionar segurança na assistência aos usuários do SUS. Nesse sentido, torna-se 
imprescindível a qualificação da força de trabalho nos componentes municipais de auditoria em relação aos aspectos burocráticos essenciais para autorização do funcionamento de serviços de fisioterapia, pois as inconformidades evidenciadas em nosso estudo ferem os princípios e diretrizes do SUS.

Ainda que ambos os serviços se referissem a estabelecimentos destinados a ações de reabilitação física, a inadequações de acessibilidade arquitetônica, estavam em desacordo com o preconizado na legislação sobre acessibilidade em serviços de saúde, o que implica sérios prejuízos para o acolhimento de pessoas com deficiência temporária ou permanente nos estabelecimentos pesquisados ${ }^{12}$.

Destacaram-se insuficiências estruturais do serviço da rede própria, em relação à ausência de espaço exclusivo destinado à administração do serviço, exames e avaliações fisioterapêuticas; banheiros separados por sexo; problemas de sinalização visual e tátil em todas as dependências e ausência de extintores de incêndio, contrariando normativas da Vigilância Sanitária estadual para serviços de fisioterapia ${ }^{13}$.

Os nossos achados sobre problemas estruturais no estabelecimento da rede própria se assemelham a problemas estruturais de outros serviços na capital baiana, sobretudo em relação à inadequação das instalações físicas ${ }^{11}$. Além disso, o espaço físico e os equipamentos eram insuficientes para realização dos atendimentos de reabilitação fisioterapêutica, assemelhando-se a achados que ratificam a fragilidade histórica de infraestrutura de serviços de reabilitação física no Brasil ${ }^{4,11}$.
Foi constatado que, em ambos os serviços, os equipamentos não atendiam parcialmente à listagem mínima da legislação estadual preconizada para o funcionamento de serviços de fisioterapia. O serviço próprio apresentou maiores problemas de equipamentos quando comparado com o serviço contratualizado, pois, além de não possuir todos os equipamentos necessários, alguns estavam em condições inadequadas de funcionamento, $o$ que comprometia a qualidade da assistência prestada.

Não evidenciamos ações de manutenção preventiva dos equipamentos de fisioterapia, já que não se comprovou a realização de estratégias. Isso aponta para a necessidade de os auditores estarem atentos à necessidade da identificação precoce de falhas dos equipamentos elétricos (sobretudo de eletrotermofototerapia), de modo que seja garantido o seu adequado funcionamento, a eficácia do tratamento e a segurança dos usuários.

A inobservância quanto ao cadastro do quantitativo de profissionais atuantes nos serviços parece indicar limitações para auditoria do SUS checar a relação entre a quantidade de fisioterapeutas e a produção ambulatorial dos serviços, interferindo diretamente sobre a averiguação do atendimento aos parâmetros assistenciais preconizados pelo Coffito. A legislação de parâmetros assistenciais fisioterapêuticos determina que, para os serviços ambulatoriais, a exemplo dos que pesquisamos, o quantitativo de atendimentos em turno de 6 horas seja de 12 usuários $^{14}$. Desse modo, o descumprimento desse padrão pode acarretar uma produção de atendimentos superior ao limite estabelecido na legislação e comprometer a qualidade da assistência prestada ao usuário. 
A inexistência do controle eletrônico da frequência dos profissionais indica que os serviços ainda preconizam o controle manual (estratégia somente presente no serviço da rede própria). $\mathrm{O}$ aprimoramento desse controle é fundamental para garantir o cumprimento da carga horária estabelecida e os parâmetros assistenciais fisioterapêuticos.

Em ambos os serviços pesquisados, alguns fisioterapeutas não portavam a cédula de registro profissional durante atendimento. A legislação do Coffito determina a obrigatoriedade do uso desse documento, durante o exercício profissional em estabelecimentos, sejam eles públicos ou privados ${ }^{15}$.

Não identificamos a realização de ações de Educação Permanente em Saúde (EPS) para os fisioterapeutas dos serviços investigados. O aumento na demanda de usuários e mudanças epidemiológicas e demográficas no estado da Bahia justificam a reorganização do processo de trabalho e das práticas de saúde nos serviços de fisioterapia do SUS, o que pressupõe a implementação de estratégias de EPS que permita problematizações a partir da prática profissional, de modo que estas possam reverberar sobre os saberes e fazeres dos fisioterapeutas, tanto na sua relação profissional-profissional quanto na profissional-usuário ${ }^{16}$.

Chamou a atenção a divergência dos serviços analisados em relação à sua integração com a CRM. Enquanto o contratualizado atua integrado à CRM, o público atua por meio de marcações manuais, por demanda espontânea e sem um fluxo estabelecido para os usuários. Esse achado evidencia que o sistema municipal de saúde não possui uma padronização na referência e contrarreferência de usuários entre o serviço próprio e contratualizado de fisioterapia ambulatorial.

A ausência de uso de protocolos assistenciais de reabilitação nos estabelecimentos que estudamos ratificou que as ações desenvolvidas carecem de uma padronização das práticas assistenciais, inclusive relativa ao processo de referência e contrarreferência, entre os serviços ambulatoriais e os serviços de fisioterapia na atenção primária à saúde. Em estudos que obtiveram achados semelhantes aos $\operatorname{nossos}^{11,17}$, a não utilização de protocolos assistenciais de reabilitação comprometia a referência e contrarreferência, dificultando a comunicação entre os pontos de atenção fisioterapêutica, com desempenho insatisfatório no encaminhamento adequado dos usuários.

A ausência de prontuários para registro da assistência no serviço contratualizado evidenciou uma grave inobservância ao que recomenda o Coffito, sobre o uso obrigatório desse instrumento nos estabelecimentos de fisioterapia públicos e privados $^{18}$. Além disso, o prontuário é um documento que permite analisar e avaliar o processo de cuidado ofertado, a produtividade, entre outros parâmetros fundamentais para tomada de decisão em auditorias do SUS ${ }^{19}$. 
Nos serviços que estudamos, a gestão de resultados era inexistente, e não havia o cumprimento das orientações técnicas do Ministério da Saúde sobre a necessidade de reavaliação clínica dos usuários, para verificação do ganho funcional e possibilidade de alta. Isso também foi confirmado pela ausência de relatórios de alta em ambos os casos.

Mesmo que tenhamos identificado o uso de "caixa de sugestões" como um possível canal de comunicação entre a gerência do serviço da rede própria e os usuários, não houve qualquer evidência de que ele fosse efetivamente utilizado para orientar as práticas gerenciais voltadas a resultados, conjecturando-se como uma aparente avaliação da percepção dos usuários.

O planejamento e a avaliação dos serviços prestados não incluíam a participação dos usuários e profissionais. Isso aponta a necessidade de que os serviços de fisioterapia do SUS possam implementar práticas gerenciais baseadas na avaliação da percepção dos usuários e profissionais, utilizando-os nas decisões tanto administrativas quanto sobre o cuidado ofertado, garantindo, desse modo, a corresponsabilização de todos na garantia do direito constitucional à saúde ${ }^{20}$.

\section{CONSIDERAÇÕES FINAIS}

Os serviços avaliados nesta pesquisa obtiveram grau incipiente de conformidade. As principais inconformidades identificadas variaram de aspectos normativos a problemas de organização e funcionamento, que podem representar riscos na prestação dos serviços e comprometer a qualidade da assistência.

O uso de dimensões e critérios bem definidos para auditoria de fisioterapia, podem tornar o processo de auditagem mais sistemático e sensível na mensuração de (in) conformidades dos serviços, colocando em destaque os pontos críticos que necessitam de intervenção preventiva, educativa e punitiva.

Recomenda-se que a gestão dos sistemas municipais e estaduais de saúde possam fortalecer seus componentes de auditoria e qualificar os atores que participam diretamente das auditorias dos serviços próprios e contratualizados do SUS, permitindo uma averiguação assertiva e suficientes para solucionar problemas na prestação do cuidado e de adequação legal.

Novos estudos sobre auditoria em fisioterapia no SUS são necessários em cenários municipais e estaduais, bem como a formulação de novos instrumentos que atualizem dimensões e critérios de análise. Isso permite refinar estratégias metodológicas nas auditorias de fisioterapia do SUS, garantindo análises cada vez mais sensíveis às necessidades de controle e fiscalização dos serviços públicos fisioterapêuticos. 


\section{REFERÊNCIAS}

1. Santos FC, Aleluia IRS, Santos IN, Moura LGF, Carvalho MA. Participação do fisioterapeuta na equipe multiprofissional de auditoria em saúde. Rev. de Administração em Saúde 2011; 13(51):95-102.

2. Brasil. Ministério da Saúde. Cadastro Nacional dos Estabelecimentos de Saúde CNES: consulta a serviços especializados [Fisioterapia-Brasil]. Brasília: Ministério da Saúde; 2017.

3. Aleluia ÍRS, Santos FC. Análise dos auditores em saúde quanto aos serviços públicos de fisioterapia no estado da Bahia. Rev. Eletrônica Gestão e Saúde 2013; 4(1):1499-1515.

4. Aleluia IRS, Sodré N, Barreto A, Júnior EPP, Santos FC. Análise da produção ambulatorial de fisioterapia no SUS, Bahia, 2008-2014. Rev. Eletrônica Gestão e Saúde 2017; 8(2):254-269.

5. Aleluia ÍRS, Santos FC. Auditoria em fisioterapia no Sistema Único de Saúde: proposta de um protocolo específico. Fisioter. mov. 2013; 26(4):725741.

6. Conselho Federal de Fisioterapia e Terapia Ocupacional. Resolução $n^{\circ}$ 416, de 19 de maio de 2012. Dispõe sobre a atuação do Fisioterapeuta como auditor e dá outras providencias. Diário Oficial da União 2018; 19 maio.

7. Aleluia ÍRS, Bahia JC, Júnior JSS, Santos FC, Santana MVS, Sousa RR, et al. Auditoria em serviços ambulatoriais de fisioterapia do SUS: proposta de instrumento. Rev. Gestão \& Saúde 2018; 9(3):365-378.

8. Mascarenhas EB. Auditoria de qualidade em fisioterapia: um instrumento para medir a satisfação do usuário. Revista Sul Americana de Auditoria em Saúde 2010; 3(1):69-96.
9. Rocha TA, Silva NC, Claudius AQ, Aamaral PV, Thumé E, Rocha JV, et al. Cadastro Nacional de Estabelecimentos de Saúde: evidências sobre a confiabilidade dos dados. Cien Saude Colet 2018; 23(1):229-240.

10. Nascimento EGQ. A importância do Cadastro Nacional de Estabelecimentos de Saúde (CNES) como instrumento na auditoria em saúde [monografia]. Curitiba (PR): Faculdades Pequeno Príncipe; 2012.

11. Santos F. Diagnóstico da situação dos serviços ambulatoriais de reabilitação física no SUS na cidade de Salvador [dissertação]. Salvador (BA): Universidade Federal da Bahia; 2013.

12. Brasil. Ministério da Saúde. Lei Federal n. 10.098 de 19 de dezembro de 2000. Estabelece normas gerais e critérios básicos para a promoção da acessibilidade das pessoas portadoras de deficiência ou com mobilidade reduzida, e dá outras providências. Diário Oficial da União 2000; 20 dez.

13. Secretaria de Saúde do Estado da Bahia. Portaria Estadual no 2.101, de outubro de 2002. Estabelece normas de Vigilância Sanitária. Bahia: Secretaria Estadual de Saúde; 2002.

14. Conselho Federal de Fisioterapia e Terapia Ocupacional. Resolução ${ }^{\circ}$ 444, de 26 de abril de 2014. Fixa e estabelece os Parâmetros Assistenciais Fisioterapêuticos nas diversas modalidades prestadas pelo fisioterapeuta. Diário Oficial da União 2014; 20 out.

15. Conselho Federal de Fisioterapia e Terapia Ocupacional. Resolução n ${ }^{\circ}$ 423, de 3 de maio de 2013. Estabelece o Código de Processo ÉticoDisciplinar da Fisioterapia e da Terapia Ocupacional. Diário Oficial da União 2013; 22 maio. 
16. Cecim RB. Educação Permanente em Saúde: desafio ambicioso e neAraújo et al cessário. Interface (Botucatu) 2005; 9(16):161-177.

17. Protassio APL, Silva PB, Lima EC, Gomes LB, Machado LS, Valença AMG. Avaliação do sistema de referência e contrarreferência do estado da Paraíba segundo os profissionais da Atenção Básica no contexto do $1^{\circ}$ ciclo de Avaliação Externa do PMAQ-AB. Saúde Debate 2014; 38:209-220.

18. Conselho Federal de Fisioterapia e Terapia Ocupacional. Resolução ${ }^{\circ}$ 414, de 23 de maio de 2012. Dispõe sobre a obrigatoriedade do registro em prontuário pelo fisioterapeuta, da guarda e do seu descarte e dá outras providências. Diário Oficial da União 2012; 23 maio.

19. Mascarenhas EB, Costa ES. Registros em Prontuários de Fisioterapia: instrumento de auditoria. Salvador. Revista Sul Americana de Auditoria em Saúde 2010; 3(1):97-122.

20. Comes Y, Trindade JS, Shimizu HE, Bargioni F, Sanchez MN, Santos LMP. Avaliação da satisfação dos usuários e da responsividade dos serviços em municípios inscritos no Programa Mais Médicos. Ciên Saúde Coletiva 2016; 21(9):27492759. 